Service social

\title{
Déterminants socioculturels et psychosociaux de l'implication dans la vie sexuelle parmi les jeunes adultes francophones du Québec
}

\author{
Joseph J. Lévy, Joanne Otis, Jean-Marc Samson, Annie Fugère et François Pilote
}

Volume 45, numéro 1, 1996

Le sexuel et le relationnel

URI : https://id.erudit.org/iderudit/706713ar

DOI : https://doi.org/10.7202/706713ar

Aller au sommaire du numéro

Éditeur(s)

École de service social de l'Université Laval

ISSN

1708-1734 (numérique)

Découvrir la revue

Citer cet article

Lévy, J. J., Otis, J., Samson, J.-M., Fugère, A. \& Pilote, F. (1996). Déterminants socioculturels et psychosociaux de l'implication dans la vie sexuelle parmi les jeunes adultes francophones du Québec. Service social, 45(1), 7-29.

https://doi.org/10.7202/706713ar
Résumé de l'article

Afin de dégager les facteurs qui interviennent dans l'entrée dans la vie sexuelle des jeunes adultes francophones du Québec, un échantillon de 2828 sujets, dont $42,3 \%$ d'hommes et 57,7 \% de femmes, inscrits dans les établissements d'enseignement collégial du Québec ont répondu à un questionnaire à remplir soi-même portant, entre autres choses, sur leurs scénarios sexuels, dont la présence d'activités génitales. Les résultats indiquent que la proportion des élèves actifs sexuellement est plus élevée chez les femmes que chez les hommes. De plus, l'entrée dans la vie sexuelle semble s'appuyer sur des considérations d'ordre sociodémographique, socioculturel et psycho-social qui renvoient à des configurations spécifiques selon le genre. Ces résultats peuvent aider à orienter les interventions sociales sur le plan de la sexualité. 


$$
A \begin{array}{llllllll} 
& R & T & I & C & L & E & S
\end{array}
$$

\title{
Déterminants socioculturels et psychosociaux de l'implication dans la vie sexuelle parmi les jeunes adultes francophones du Québec
}

\author{
Joseph J. LÉVY \\ Professeur \\ Joanne OTIS \\ Professeure \\ Jean-Marc SAMSON \\ Professeur \\ Annie FUGÈRE \\ Étudiante à la maîtrise \\ François PILOTE \\ Étudiant à la maîtrise \\ Département de sexologie \\ Université du Québec à Montréal
}

\section{INTRODUCTION}

Dans les sociétés postindustralisées contemporaines, les conduites sexuelles des adolescents et des jeunes adultes prennent place dans un contexte marqué par une permissivité sexuelle plus affirmée et l'accès facile à une contraception efficace, même

Cette recherche a été subventionnée par le Programme national de recherche et de développement en matière de santé (PNRDS). 
si des doubles messages continuent d'entourer le rapport à la sexualité dans ces groupes d'âge. Ce contexte a néanmoins contribué à accroître de façon significative le pourcentage des jeunes qui ont des relations coïtales et ce, à un âge de plus en plus jeune. Dans le contexte québécois marqué par une modernisation très rapide des conduites sexuelles chez les adolescents (Lévy et Sansfaçon, 1994), on constate que, pour les élèves d'établissements d'enseignement collégial, ce pourcentage passe d'environ $50 \%$ à la fin des années 70 à des chiffres variant selon les recherches entre $58 \%$ et $84 \%$ pour la fin des années 80 et le début des années 90 (Otis, 1996), l'âge moyen se situant à 16 ans. Cette situation n'est pas sans avoir des répercussions sur le plan de l'intervention sociale ou préventive auprès de ces populations dans la mesure où elle peut s'accompagner de pratiques sexuelles à risque par rapport aux MTS et au sida. Aussi, la compréhension des facteurs qui interviennent dans la décision de devenir sexuellement actif peut aider à mieux cibler les interventions et à mieux préparer les jeunes à ce passage.

L'implication sexuelle (définie ici comme l'accès à une activité génitale dans une relation interpersonnelle) des adolescents et des jeunes adultes (distinction qui n'est pas toujours précisée dans les recherches) a surtout été étudiée dans le contexte de la société américaine. Quatre ensembles de facteurs, sociodémographiques, socioéconomiques, socioculturels et psychosociaux (individuels, interpersonnels et normatifs) ont été ainsi dégagés à ce sujet (Chilman, 1983; Christopher et Roosa, 1991 ; Gullotta, Adams et Montmayer, 1993).

Sur le plan sociodémographique, les probabilités d'avoir des relations sexuelles s'accroissent avec l'âge (Zelnick et Shah, 1983 ; Dorius, Heaton et Steffen, 1993). Les filles commencent leur vie sexuelle à un âge plus tardif que les garçons (Zelnick et Shah, 1983 ; Dorius, Heaton et Steffen, 1993). Ces écarts suggèrent que la socialisation sexuelle continue d'obéir à un double standard qui favorise l'expression de la sexualité masculine et oriente les scénarios sexuels.

L'implication sexuelle est aussi modulée par des facteurs socioéconomiques qui peuvent orienter l'intervention sociale. Ainsi, les adolescents qui proviennent de milieux économiquement défavorisés tendent à s'engager plus rapidement dans les activités coïtales (Zelnik et Kantner, 1980; Chilman, 1980 ; Moore, 1990), tout comme ceux dont la scolarité des parents est faible (Russ-Eft, Sprenger et Beever, 1979; Zelnik, Kantner 
et Ford, 1981; Dorius, Heaton et Steffen, 1993). Le mode de vie urbain favoriserait l'entrée dans la vie sexuelle (Zelnik et Kantner, 1980 ; Dryfoos, 1982), mais d'autres études montrent que les adolescentes des classes moyennes qui vivent en banlieue sont plus enclines à être conservatrices sur le plan sexuel (Forste et Heaton, 1988).

Sur le plan socioculturel, l'identité ethnique semble aussi intervenir. Ainsi, plus d'Afro-Américains que de Caucasiens ou d'Hispaniques sont sexuellement actifs (Zelnik et Kantner, 1980; Dorius, Heaton et Steffen, 1993) et ce, pour tous les groupes d'âge (Forste et Heaton, 1988), ce qui pourrait s'expliquer par des variations dans les valeurs sexuelles et familiales, associées à des conditions économiques difficiles.

Les répercussions des structures et des valeurs familiales sur la vie sexuelle des adolescents et des jeunes adultes ont aussi été cernées. Ainsi, lorsque ces derniers vivent dans une famille biparentale où la mère reste au foyer, ils ont tendance à retarder le début des relations coïtales (Forste et Heaton, 1988; Miller et Bingham, 1989; Moore, 1990; Flewelling et Bauman, 1990; Young, Jensen, Olsen et Cundick, 1991; Dorius, Heaton et Steffen, 1993). Les modes de socialisation et de communication dans la famille ne sont pas non plus étrangers à ces stratégies, même si les recherches ne sont pas toujours convergentes. Ainsi, l'entrée dans l'activité coïtale des adolescentes semble être moins fréquente lorsque les parents ont des valeurs familiales traditionnelles, mais discutent néanmoins de sujets sexuels (Moore, Simms et Betsey, 1986). Il en est de même lorsque les parents exercent une supervision serrée sur leurs rendez-vous (Miller, McCoy, Olsen et Wallace, 1986). Par contre, dans le cas où les parents ont des attitudes libérales ou lorsqu'il s'agit de parents conservateurs qui ne discutent pas de sexualité, les adolescentes ont des activités coïtales plus rapidement (Fisher, 1989; Weinstein et Thornton, 1989). La qualité des relations avec les parents affecte aussi l'entrée dans la vie sexuelle (Whitbeck, Hoyt, Miller et Kao, 1992), mais de façon différente selon le genre. Chez les garçons, une insatisfaction au niveau des relations familiales les pousserait davantage à adopter diverses conduites à risque autres que sexuelles (alcool, par exemple). Devant cette insatisfaction les filles, par contre, s'orienteraient davantage vers la quête de relations affectives où la dimension sexuelle intervient. Lorsque la communication mère-fille est bonne, les filles sont moins enclines à devenir coïtalement actives (Miller, Card, 
Paikoff, et Peterson, 1992), alors que pour les garçons une meilleure communication avec leur père augmente les probabilités d'avoir des relations sexuelles (Kahn, Smith, et Roberts, 1984). Plusieurs recherches, par contre, ne rapportent pas d'associations significatives entre ces deux dimensions (Furstenberg, Moore et Peterson, 1985 ; Furstenberg, Herceg-Baron, Shea et Webb, 1986; Treboux et Busch-Rossnagel, 1990). En lien avec le contexte familial, les adolescents peuvent se conformer aux modèles offerts par leur fratrie. Si elle est active sexuellement, ils le deviendront aussi plus rapidement (Rodgers et Rowe, 1988). De même, les pairs, dont l'influence dépasse souvent celle des parents, ce qui correspond à un stade du développement normal chez les adolescents, peuvent exercer une pression sociosexuelle (Harris, 1986 ; Billy et Udry, 1985 ; Rodgers et Rowe, 1990), en particulier sur les filles (Miller, Card, Paikoff et Peterson, 1992), et les pousser à commencer leur vie coïtale plus rapidement.

Sur le plan psychosocial, plusieurs facteurs interviennent sur ces conduites. Ainsi, les individus plus religieux et plus pratiquants sont plus enclins à retarder leur entrée dans la vie sexuelle (Chilman,1980; Thornton et Camburn, 1987; White et DeBlassie, 1992; Delamater, 1981; Forste et Heaton, 1988; Miller et Olsen, 1988 ; Sugar, 1990, Moore, 1990), en particulier parmi les garçons (Thornton et Camburn, 1987). Il en est de même chez ceux pour qui le succès scolaire est important (Stewart, 1987 ; Hayes, 1987 ; Hofferth, 1987 ; Moore, 1990). Le type de foyer de contrôle et une forte estime de soi ont été associés à l'âge d'entrée dans la vie sexuelle, mais les résultats ne sont pas toujours convergents (Strouse et Fabes, 1987 ; Miller, Christensen et Olsen, 1987). Une perspective traditionnelle quant aux rôles sexuels défavoriserait l'implication coïtale (Cverkovich, Grote, Lieberman, et Miller, 1978; Thornton et Camburn, 1987 ; Miller et Olson, 1988 ; Christopher et Roosa, 1991), de même qu'un niveau de culpabilité sexuelle élevé (Propper et Brown, 1986). Le niveau de peur lié aux infections liées aux MTS et au sida ne semble pas intervenir sur les décisions d'avoir des relations sexuelles, contrairement à la peur de la grossesse (Jedlicka et Robinson, 1987). Par contre, les adolescents et les jeunes adultes qui ont reçu une éducation sexuelle ou des informations sur la sexualité et la reproduction sont aussi portés à retarder le début des relations coïtales (Cverkovich et Grote, 1980 ; Rosen, Herskovitz et Stack, 1980 ; Zelnik et Kim, 1982 ; Forste et Heaton, 1988). 
Sur le plan interpersonnel, les modes de fréquentation affectent l'entrée dans la vie sexuelle en augmentant les probabilités des relations (Dorius, Heaton et Steffen, 1993), mais les facteurs en jeu diffèrent selon le genre. Ainsi, les filles tendent à associer leurs relations sexuelles à un contexte amoureux et à un engagement émotif significatif, contrairement aux garçons (Carroll, Volk et Hyde, 1985 ; Christopher et Cate, 1984). Les conceptions des liens entre sexualité et amour peuvent aussi intervenir. Les femmes qui considèrent que l'amour et la sexualité sont deux aspects associés ont tendance à commencer leur vie sexuelle plus tardivement que celles qui séparent ces deux sphères (Rosen et Herskovitz, 1982), une perspective que semblent adopter plus volontiers les garçons, ce qui expliquerait leur précocité plus grande. Le degré d'intimité et l'importance du sentiment amoureux dans la relation joueraient aussi un rôle non négligeable dans l'entrée dans la vie sexuelle (Christopher et Cate, 1984, 1988), de même que les modalités d'influence ou de coercition qu'exercent les partenaires (Christopher et Roosa, 1991).

L'approche statistique multivariée (Smith et al., 1993) a permis de préciser les déterminants les plus significatifs dans la décision de s'impliquer coïtalement. Ainsi, ceux qui n'étaient pas actifs coïtalement étaient plus jeunes, d'origine caucasienne, avaient reçu plus d'heures d'éducation sexuelle, ressentaient plus de culpabilité à l'égard de la sexualité, percevaient un risque plus faible, considéraient que les normes des pairs étaient favorables à la continence, avaient une croyance élevée quant à leur efficacité personnelle, décidaient plus fréquemment de leurs actions en fonction de principes religieux, n'étaient pas engagés dans une relation affective stable et avaient eu peu ou pas de discussions avec un partenaire sur le VIH ou le sida.

Cet ensemble de recherches indiquent que l'entrée dans l'activité sexuelle génitale obéit à des configurations complexes, mais celles-ci sont bien moins connues pour le Québec. Ainsi un engagement plus rapide dans l'activité génitale se retrouve chez les Québécois francophones (Santé Québec, 1992) et est associé à une résidence urbaine (Table de concertation sur la santé et la sexualité de la commission scolaire La Neigette, 1995) et à une faible pratique religieuse (Santé Québec, 1992). Le fait de vivre ailleurs que chez ses parents, en particulier avec la ou le partenaire amoureux, contribue à cette décision. Les motivations qui peuvent jouer dans les décisions de retarder l'entrée dans la vie 
sexuelle ont aussi été cernées par Otis, Godin et Lambert (1993). Dans leur étude, sept variables expliquent $55 \%$ de la variation totale de l'intention de retarder l'entrée dans la vie sexuelle parmi un échantillon probabiliste d'adolescents de la $1^{\text {re }}$ à la $5^{\mathrm{e}}$ secondaire. On retrouve ainsi une forte croyance que la continence est hautement valorisée par les pairs, une norme morale élevée et une attitude positive à l'égard de ce comportement, de même que l'impression d'être davantage capable de reporter le début de cette activité. Cette intention du report est très modérée, particulièrement chez les garçons les plus âgés. Chez les filles, les principaux avantages perçus relèvent du désir de faire un apprentissage graduel de la sexualité, alors que chez les garçons le respect est la principale raison avancée. Les premières perçoivent une pression pour retarder leur entrée dans la vie sexuelle de la part des figures d'autorité (parents, professeurs, etc.), alors que les garçons et leurs partenaires amoureux les poussent, au contraire, à commencer leur vie coïtale plus rapidement.

En résumé, ces recherches montrent que les stratégies liées à l'implication dans les activités sexuelles dépendent de variables complexes dont les configurations et les déterminants sont encore mal cernés pour les populations de jeunes adultes québécois. Ainsi, afin de mieux intervenir sur le plan préventif, il serait important de dégager les niveaux d'implication sexuelle dans ce groupe d'âge, de même que les facteurs d'ordre sociodémographique et psychosocial sous-jacents à ces stratégies sexuelles, en tenant compte en particulier du type de résidence, rurale ou urbaine. Dans cette perspective, nous présenterons ici les résultats d'une recherche menée auprès de jeunes adultes fréquentant les établissements francophones d'enseignement collégial du Québec.

\section{MÉTHODOLOGIE}

\section{Les sujets}

Au cours de l'année 1995, les 65 collèges francophones publics et privés du Québec ont été sollicités afin de s'associer à une recherche portant sur les risques face au sida dans la population étudiante (Lévy, Otis et Samson, 1996). Six d'entre eux ont refusé leur collaboration. La population étudiée était composée de l'ensemble des élèves inscrits à temps plein dans le profil général ou technique. Une stratégie d'échantillonnage stratifié 
en grappe à une phase (par groupe-cours) a été privilégiée afin de rejoindre $2 \%$ de la population des collèges de la région de Montréal et $3 \%$ de la population des collèges dans les autres régions. Dans la majorité des collèges, le recrutement s'est fait en rejoignant les élèves dans leurs cours obligatoires de français ou de philosophie, mais, dans certains cas, les élèves ont été approchés dans d'autres cours. Après élimination des questionnaires incomplets ou mal remplis, l'échantillon final a été défini. Il se compose de 2828 sujets, dont $42,3 \%$ d'hommes et $57,7 \%$ de femmes. Parmi eux, 6,3\% avaient eu des relations de type homosexuel et ont été comptés parmi les répondants sexuellement actifs. Sur le plan de la répartition géographique, 45,3\% étudient dans la région de Montréal et de Québec et 54,7\% dans les autres régions du Québec. Quant à la concentration d'études, $58,3 \%$ sont inscrits dans le profil général et $41,7 \%$ dans la filière technique ; $37,7 \%$ sont inscrits en sciences humaines, $18,5 \%$ en sciences administratives, $17,9 \%$ en sciences physiques, $17 \%$ en sciences biologiques. Parmi ces étudiants, 75,7\% avaient déjà eu au moins une relation coïtale, l'âge moyen à la première relation étant de 15,8 ans et de 16,1 à la seconde.

\section{Le questionnaire}

Un questionnaire élaboré aux fins de l'étude a été auto-administré dans un contexte d'anonymat et sur une base volontaire. Il comprenait plusieurs sections qui couvraient notamment les dimensions sociodémographiques (genre, origine, langue maternelle, religion pratiquée, collège fréquenté, etc.), les traits de personnalité, les motivations des prises de risques, l'égalité dans les rôles sexuels, les attitudes et les perceptions du contrôle concernant l'usage du condom, l'évaluation de la peur et des risques par rapport à la grossesse, aux MTS et au sida et finalement le statut sexuel. Les diverses échelles ont été soumises à l'analyse en composante principale de manière à démontrer la validité des construits. Les énoncés appartenant à un même facteur ont par la suite fait l'objet d'analyses de fiabilité pour en vérifier la consistance interne et déterminer la valeur de l'alpha de Cronbach. Pour plus de lisibilité, ces scores factoriels ont tous été convertis en scores $T$, c'est-à-dire qu'ils ont été arithmétiquement ramenés d'une moyenne et d'un écart-type de zéro à une moyenne de 50 et à un écart-type de 10. Plusieurs analyses statistiques ont été utilisées (distribution de fréquences, tableaux de contingence et tests $t$ de Student). En plus de ces analyses bivariées, des analyses 
de régression logistique ont permis de dégager les principaux déterminants qui interviennent dans l'entrée dans la vie sexuelle des collégiens. Les données recueillies ont été analysées à l'aide du progiciel SPSS 4,0 pour UNIX.

\section{RÉSULTATS}

\section{Déterminants sociodémographiques et socioculturels}

Comme le montre le tableau 1 construit à partir des analyses bivariées, on peut dégager de nombreuses associations significatives entre les variables sociodémographiques, socioculturelles et l'entrée ou non dans la vie sexuelle.

Sur le plan sociodémographique, on constate que les garçons sont plus nombreux à ne pas avoir eu de relations génitales que les filles. L'âge intervient aussi, puisque les sujets plus âgés sont plus nombreux à être actifs sexuellement. Les étudiants en sciences humaines et lettres et ceux fréquentant les collèges privés sont significativement moins nombreux à être actifs sexuellement, tout comme ceux qui sont inscrits en première année de cégep. Ceux qui ont une occupation sont plus impliqués sexuellement et, dans ce groupe, ce pourcentage est plus élevé chez ceux qui ont plus d'heures de travail. L'organisation familiale intervient aussi sur leur statut sexuel. Ainsi, le pourcentage des étudiants qui n'ont pas eu activités sexuelles est plus élevé parmi ceux qui vivent avec leurs deux parents. Le niveau socioéconomique des parents et le fait que l'un ou l'autre travaille ne modulent pas l'entrée dans la vie sexuelle. Par contre, lorsque les deux parents travaillent, le pourcentage de ceux qui sont impliqués sexuellement tend à augmenter significativement. Une tendance inverse se retrouve parmi les étudiants nés ailleurs qu'au Québec, qui ont une autre langue maternelle que le français et qui continuent de l'utiliser dans leurs rapports familiaux, de même que chez les plus croyants et les plus pratiquants.

Le recours à la régression logistique, qui permet de préciser les facteurs les plus significatifs (tableau 2), montre que la nonimplication sexuelle est plus forte parmi les garçons, les répondants vivant avec leurs deux parents et les plus pratiquants sur le plan de la pratique religieuse. Les répondants actifs sexuellement, pour leur part, sont proportionnellement plus nombreux parmi les répondants plus âgés, parmi les étudiants inscrits en 
Tableau 1

Variables démographiques et socioculturelles différenciant les élèves sexuellement impliqués ou non

$(\mathrm{N}=2828)$

\begin{tabular}{|c|c|c|c|}
\hline Variables & $\begin{array}{c}\text { Non impliqués } \\
(\mathrm{N}=686) \\
\% \\
\end{array}$ & $\begin{array}{c}\text { Impliqués } \\
(\mathrm{N}=2142) \\
\%\end{array}$ & Valeur de $p$ \\
\hline \multicolumn{4}{|l|}{ Genre } \\
\hline Hommes & 29,9 & 70,1 & \multirow[t]{2}{*}{$* * *$} \\
\hline Femmes & 20,1 & 79,9 & \\
\hline \multicolumn{4}{|l|}{ Âge } \\
\hline $16-18$ ans & 29,3 & 70,7 & \multirow[t]{2}{*}{$* * *$} \\
\hline $19-21$ ans & 17,6 & 82,4 & \\
\hline \multicolumn{4}{|l|}{ Concentration } \\
\hline Sciences pures & 27,7 & 72,3 & \multirow[t]{2}{*}{$* * *$} \\
\hline Sciences humaines & 20,6 & 79,4 & \\
\hline \multicolumn{4}{|l|}{ Type de collège } \\
\hline Public & 22,8 & 77,2 & \multirow[b]{2}{*}{$\star \star \star \star ~$} \\
\hline Privé & 40,0 & 60,0 & \\
\hline \multicolumn{4}{|l|}{ Niveau } \\
\hline $1^{\text {re }}$ collégiale & 30,0 & 70,0 & \multirow[t]{2}{*}{$* * *$} \\
\hline $2^{\mathrm{e}}$ et $3^{\mathrm{e}}$ collégiale & 20,0 & 80,0 & \\
\hline \multicolumn{4}{|l|}{ Statut d'emploi } \\
\hline Sans travail & 31,0 & 69,0 & \multirow[t]{2}{*}{$* * *$} \\
\hline Travail & 17,7 & 82,3 & \\
\hline \multicolumn{4}{|l|}{ Parmi les élèves qui travaillent: } \\
\hline Heures travaillées : 1 à 9 & 25,3 & 74,7 & \multirow[t]{2}{*}{$* * *$} \\
\hline Heures travaillées : 10 et plus & 15,6 & 84,4 & \\
\hline \multicolumn{4}{|l|}{ Statut de la famille } \\
\hline Vivre avec les deux parents & 29,1 & 70,9 & \multirow[t]{2}{*}{$* * *$} \\
\hline Vivre avec d'autres & 16,5 & 83,5 & \\
\hline \multicolumn{4}{|l|}{ Emploi des parents } \\
\hline Un parent travaille & 27,9 & 72,1 & \multirow[t]{2}{*}{$* \star *$} \\
\hline Deux parents travaillent & 21,6 & 78,4 & \\
\hline \multicolumn{4}{|l|}{ Lieu de naissance } \\
\hline Québec & 23,7 & 76,3 & \multirow[b]{2}{*}{ * } \\
\hline Hors Québec & 33,1 & 66,9 & \\
\hline \multicolumn{4}{|l|}{ Langue maternelle } \\
\hline Français & 23,3 & 76,7 & \multirow[b]{2}{*}{$* * *$} \\
\hline Autre & 44,4 & 55,6 & \\
\hline \multicolumn{4}{|l|}{ Langue parlée à la maison } \\
\hline Français & 23,6 & 76,4 & \multirow[b]{2}{*}{$* * *$} \\
\hline Autre & 35,2 & 64,8 & \\
\hline \multicolumn{4}{|l|}{ Religion } \\
\hline Peu croyant & 21,8 & 78,2 & \multirow[t]{2}{*}{$* * *$} \\
\hline Très croyant & 27,4 & 72,6 & \\
\hline Pratique religieuse & & & \\
\hline Faible & 22,0 & 78,0 & $\star \star \star *$ \\
\hline Forte & 37,7 & 62,3 & \\
\hline
\end{tabular}

*** p\# $0,0001 \quad$ ** p\# $0,001 \quad$ * $p \# 0,01$ 


\section{TABleau 2}

Déterminants démographiques et socioculturels de l'implication sexuelle chez les élèves du collégial

\begin{tabular}{|c|c|c|c|}
\hline Variables & $\begin{array}{c}\text { Échantillon } \\
\text { total } \\
(\mathrm{N}=2828)\end{array}$ & $\begin{array}{l}\text { Garçons } \\
(\mathrm{N}=1196)\end{array}$ & $\begin{array}{c}\text { Filles } \\
(\mathrm{N}=1632)\end{array}$ \\
\hline & RC $\quad$ [IC $95 \%$ \% & RC [IC $95 \%$ ] & RC [IC $95 \%$ ] \\
\hline Genre & $0,55[0,32-0,66]$ & - & - \\
\hline Âge & $1,56[1,25-1,93]$ & $1,43[1,06-1,92]$ & $1,77[1,34-2,34]$ \\
\hline Niveau scolaire & $1,30[1,06-1,60]$ & $1,50[1,13-2,00]$ & $\longrightarrow$ \\
\hline $\begin{array}{l}\text { Travail pendant } \\
\text { les études }\end{array}$ & $2,00[1,66-2,34]$ & $1,68[1,29-2,18]$ & $2,42[1,84-3,17]$ \\
\hline $\begin{array}{l}\text { Vivre avec } \\
\text { les deux parents }\end{array}$ & $0,50[0,41-0,62]$ & $0,61[0,46-0,81]$ & $0,44[0,33-0,59]$ \\
\hline Langue maternelle & $2,09[1,41-3,08]$ & $\longrightarrow$ & $3,36[2,02-5,59]$ \\
\hline Pratique religieuse & $0,52[0,41-0,66]$ & $0,66[0,47-0,95]$ & $0,44[0,32-0,60]$ \\
\hline Cégep public & $2,04[1,52-2,73]$ & $1,94[1,30-3,12]$ & $1,99[1,36-2,91]$ \\
\hline $\begin{array}{l}\text { Profil scolaire } \\
\text { général }\end{array}$ & 一- & - & $0,75[0,56-0,98]$ \\
\hline $\begin{array}{l}\text { Ajustement du modèle } \\
\% \text { de cas }\end{array}$ & $\begin{array}{l}\chi^{2}=285,84 \\
\mathrm{dl}=8 \mathrm{p}<0,0001\end{array}$ & $\begin{array}{l}\chi^{2}=74,87 \\
\mathrm{dl}=7 \mathrm{p}<0,0001\end{array}$ & $\begin{array}{l}\chi^{2}=200,55 \\
\mathrm{dl}=7 \mathrm{p}<0,0001\end{array}$ \\
\hline correctement classifiés & $76,10 \%$ & $74,87 \%$ & $80,58 \%$ \\
\hline
\end{tabular}

$2^{\mathrm{e}}$ et $3^{\mathrm{e}}$ collégiale et parmi ceux dont la langue maternelle est le français. Davantage d'entre eux travaillent pendant leurs études et fréquentent un collège public (cégep). Le genre étant un prédicteur significatif, nous avons effectué une analyse séparée pour les garçons et les filles afin de dégager les déterminants spécifiques. Les résultats indiquent un profil légèrement différent entre eux (tableau 2). Ainsi, parmi les garçons, la langue maternelle n'est pas un prédicteur significatif, contrairement à ce que l'on observe chez les filles. Chez ces dernières, le niveau scolaire n'intervient pas, à l'opposé de ce que l'on constate chez les garçons. Les filles non impliquées sexuellement seraient aussi davantage inscrites dans le profil technique plutôt que général, un facteur qui ne joue pas chez les garçons.

\section{Déterminants psychosociaux}

Comme le montre le tableau 3, plusieurs associations significatives entre les facteurs psychosociaux et l'implication sexuelle sont présentes. 
Les élèves non impliqués sexuellement sont moins enclins à exprimer des traits de personnalité comme l'empathie comparativement aux répondants qui ont déjà eu des activités sexuelles. La recherche des sensations fortes est moins élevée dans le premier groupe, tout comme les motivations intrinsèques à prendre des risques. Sur le plan des conceptions de la sexualité, les répondants qui n'ont pas eu d'activités sexuelles épousent davantage une vision où domine le partage; l'égalité dans les rôles sexuels est aussi plus prononcée.

Les perceptions du contrôle de la sexualité obéissent également à des modalités différentes. Les répondants non impliqués sexuellement se disent davantage en contrôle de leurs pulsions sexuelles, contrairement aux élèves sexuellement actifs qui, dans les stratégies de contrôle de leur sexualité, accordent plus

\section{TABLEAU 3}

Variables psychosociales différenciant les élèves sexuellement impliqués ou non

$(N=2828)$

\begin{tabular}{|c|c|c|c|}
\hline Variables & $\begin{array}{c}\text { Non impliqués } \\
(\mathrm{N}=686) \\
\mathrm{M}\end{array}$ & $\begin{array}{c}\text { Impliqués } \\
(\mathrm{N}=2142) \\
\mathrm{M} \\
\end{array}$ & Valeur de $p$ \\
\hline $\begin{array}{l}\text { Traits de personnalité } \\
\text { Empathie }\end{array}$ & 48,5 & 50,5 & $* * *$ \\
\hline $\begin{array}{l}\text { Quête de sensations fortes } \\
\text { Sensations fortes } \\
\text { Motivations : intrinsèques }\end{array}$ & $\begin{array}{l}48,3 \\
48,5\end{array}$ & $\begin{array}{l}50,5 \\
50,5\end{array}$ & $* * *$ \\
\hline $\begin{array}{l}\text { Égalité dans les rôles sexuels } \\
\text { Attitudes et perceptions } \\
\text { de contrôle vis-à-vis de la sext }\end{array}$ & Ialité & 50,7 & $* * *$ \\
\hline $\begin{array}{l}\text { Attitudes: partage } \\
\text { Contrôle : pulsions sexuelles } \\
\text { Contrôle : plaisir sexuel }\end{array}$ & $\begin{array}{l}48,6 \\
50,9 \\
44,9\end{array}$ & $\begin{array}{l}50,5 \\
49,7 \\
51,6\end{array}$ & $\begin{array}{r}* * * \\
* \\
* * *\end{array}$ \\
\hline $\begin{array}{l}\text { Attitudes et perceptions } \\
\text { de contrôle vis-à-vis du condo }\end{array}$ & & & \\
\hline $\begin{array}{l}\text { Attitudes : tolérable } \\
\text { Contrôle : relation } \\
\text { Contrôle : gêne }\end{array}$ & $\begin{array}{l}52,7 \\
52,6 \\
44,5\end{array}$ & $\begin{array}{l}49,1 \\
49,2 \\
51,8\end{array}$ & $\begin{array}{l}* * * \\
* * * \\
* * *\end{array}$ \\
\hline $\begin{array}{l}\text { Réactions devant une grossess } \\
\text { Peur } \\
\text { Risques }\end{array}$ & $\begin{array}{l}4,1 \\
1,3\end{array}$ & $\begin{array}{l}3,9 \\
1,9\end{array}$ & * \\
\hline $\begin{array}{l}\text { Réactions devant une MTS } \\
\text { ou le sida } \\
\text { Peur } \\
\text { Risques }\end{array}$ & $\begin{array}{l}50,8 \\
47,1\end{array}$ & $\begin{array}{l}49,7 \\
50,9\end{array}$ & * \\
\hline
\end{tabular}




\section{Tableau 4}

Déterminants psychosociaux de l'implication sexuelle chez les élèves du collégial

\begin{tabular}{|c|c|c|c|}
\hline Variables & $\begin{array}{c}\text { Échantillon } \\
\text { total } \\
(\mathrm{N}=2828)\end{array}$ & $\begin{array}{l}\text { Garçons } \\
(\mathrm{N}=1196)\end{array}$ & $\begin{array}{c}\text { Filles } \\
(\mathrm{N}=1632)\end{array}$ \\
\hline & RC [IC $95 \%$ ] & RC [IC $95 \%$ ] & RC $\quad\left[\begin{array}{lll}\text { IC } & 95 \%\end{array}\right]$ \\
\hline Genre & $0,38[0,31-0,47]$ & -_. & - \\
\hline $\begin{array}{l}\text { Trait de personnalité: } \\
\text { domination }\end{array}$ & $1,02[1,00-1,03]$ & $1,02[1,01-1,04]$ & -- \\
\hline $\begin{array}{l}\text { Quête de sensations } \\
\text { fortes }\end{array}$ & $1,02[1,01-1,03]$ & $1,04[1,03-1,06]$ & - \\
\hline $\begin{array}{l}\text { Attitudes à l'égard de } \\
\text { la sexualité: partage }\end{array}$ & 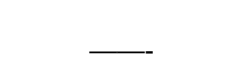 & $\longrightarrow$ & $1,02[1,00-1,03]$ \\
\hline $\begin{array}{l}\text { Égalité dans } \\
\text { les rôles sexuels }\end{array}$ & $1,02[1,01-1,03]$ & $1,02[1,00-1,03]$ & $1,02[1,01-1,04]$ \\
\hline $\begin{array}{l}\text { Perceptions du } \\
\text { contrôle de } \\
\text { la sexualité : plaisir }\end{array}$ & $1,04[1,03-1,05]$ & $1,04[1,02-1,05]$ & $1,04[1,03-1,06]$ \\
\hline $\begin{array}{l}\text { Perceptions } d u \\
\text { contrôle de } \\
\text { la sexualité : volonté }\end{array}$ & $0,99[0,98-1,00]$ & $0,98[0,97-0,99]$ & - \\
\hline $\begin{array}{l}\text { Attitudes à l'égard } \\
\text { du condom : tolérable }\end{array}$ & $0,97[0,96-0,98]$ & -- & $0,95[0,93-0,97]$ \\
\hline $\begin{array}{l}\text { Perceptions du contrôle } \\
\text { du condom: } \\
\text { dans la relation }\end{array}$ & $0,97[0,96-0,98]$ & $0,97[0,95-0,99]$ & $0,96[0,95-0,98]$ \\
\hline $\begin{array}{l}\text { Perceptions du contrôle } \\
\text { du condom : gêne }\end{array}$ & $1,06[1,05-1,07]$ & $1,05[1,04-1,07]$ & $1,06[1,04-1,08]$ \\
\hline $\begin{array}{l}\text { Être confronté }(e) \text { à } \\
\text { une grossesse : peur }\end{array}$ & $0,87[0,81-0,95]$ & - & $0,83[0,74-0,94]$ \\
\hline $\begin{array}{l}\text { Etre confronté(e) à } \\
\quad \text { une grossesse : risques }\end{array}$ & - & $1,87[1,54-2,27]$ & $2,38[1,88-3,03]$ \\
\hline $\begin{array}{l}\text { Contracter une MTS } \\
\text { ou le sida: peur }\end{array}$ & $2,00[1,72-2,34]$ & $0,98[0,97-0,99]$ & -- \\
\hline $\begin{array}{c}\text { Contracter une MTS } \\
\text { ou le sida: risques }\end{array}$ & $1,02[1,01-1,04]$ & - & $1,03[1,01-1,05]$ \\
\hline $\begin{array}{l}\text { Ajustement du modèle } \\
\% \text { de cas } \\
\text { correctement classifiés }\end{array}$ & $\begin{array}{l}\chi^{2}=728,34 \\
d l=12 p<0,0001\end{array}$ & $\begin{array}{l}\chi^{2}=302,63 \\
\mathrm{dl}=9 \mathrm{p}<0,0001 \\
78,09 \%\end{array}$ & $\begin{array}{l}\chi^{2}=428,38 \\
\mathrm{dl}=97 \mathrm{p}<0,0001 \\
83,76 \%\end{array}$ \\
\hline
\end{tabular}


d'importance au plaisir. Les attitudes à l'égard du préservatif présentent aussi des différences significatives. Pour les sujets non actifs, le préservatif est plus acceptable dans la relation sexuelle, alors que sur le plan du contrôle sur son usage ils affirment être davantage capables de l'utiliser dans la relation sexuelle (même en dépit de certaines situations comme la passion, le fait d'être avec un partenaire régulier, etc.) et sont moins gênés d'y avoir recours. La perception des risques de faire face à une grossesse ou de contracter une MTS ou le sida est plus élevée chez les répondants impliqués sexuellement ; par contre, la peur de contracter une MTS ou le sida semble avoir influencé plus fortement leurs habitudes sexuelles. Les autres répondants, pour leur part, semblent plus préoccupés par la peur d'une éventuelle grossesse que par les autres dangers.

L'analyse des déterminants psychosociaux qui interviennent dans l'implication sexuelle à partir de la régression logistique (tableau 4) montre que la majorité des variables entrent dans le modèle final. En sont exclues l'empathie, les motivations intrinsèques à prendre des risques, une conception de la sexualité basée sur le pouvoir, la perception du contrôle des pulsions sexuelles et la perception de la nécessité du préservatif. Le genre continuant d'être un prédicteur significatif, nous avons effectué une analyse séparée pour les garçons et les filles (tableau 4).

Certains facteurs préalablement identifiés semblent distinguer les répondants impliqués sexuellement de ceux qui ne le sont pas, certains jouant uniquement chez les garçons ou chez les filles. Chez les garçons, les sexuellement actifs se distinguent par une personnalité plus dominante et une quête plus élevée de sensations fortes. Ils perçoivent que leur contrôle quant au respect de leurs demandes dans la sphère sexuelle est faible et, contrairement à ce qui est observé dans l'échantillon total, ils sont moins nombreux que les garçons non impliqués sexuellement à rapporter que la peur de contracter une MTS ou le sida a changé leurs habitudes sexuelles.

Les jeunes filles non actives sexuellement ont, pour leur part, des attitudes à l'égard de la sexualité orientées davantage vers le partage, mais celles à l'égard de l'usage du condom sont plus négatives. La peur d'être confrontées à une grossesse semble avoir moins influencé leurs habitudes sexuelles, mais, par contre, les risques d'être confrontées à une grossesse et de contracter une MTS ou le sida sont perçus comme plus élevés. 


\section{DISCUSSION}

À partir de ces résultats, il est possible de dégager les facteurs les plus déterminants qui entourent l'implication sexuelle parmi les élèves francophones des établissements d'enseignement collégial de l'ensemble du Québec. On peut noter tout d'abord que les trois quarts des élèves ont déjà eu des relations sexuelles, ce qui confirme la progression du pourcentage des jeunes adultes qui sont actifs sexuellement, même si une minorité continue de maintenir un statut sexuel de continence. Celle-ci s'explique par plusieurs facteurs.

\section{Déterminants sociodémographiques et socioculturels}

Les déterminants sociodémographiques et socioculturels recoupent en grande partie ceux dégagés par d'autres recherches. Il faut noter cependant un résultat qui va à l'encontre de ceux rapportés dans l'ensemble des autres recherches (voir Chilman, 1983 et Christopher et Roosa, 1991 pour une revue de la littérature) qui indiquent toutes que les garçons, pour un même âge, sont plus enclins à être sexuellement actifs, alors que notre recherche montre que plus de garçons (10\%) que de filles se retrouvent dans le groupe des répondants non impliqués sexuellement. Cet écart pourrait être attribué à des distorsions dans l'échantillon lié à un biais de sélection. Sans totalement écarter cette éventualité, il faut cependant noter qu'une analyse plus fine de la répartition des garçons et des filles selon leur statut sexuel par région ne montre aucune différence significative, sauf dans la région du Saguenay - Lac-Saint-Jean, où les filles impliquées sexuellement sont proportionnellement plus nombreuses et plus jeunes qu'ailleurs. Ce résultat suggérerait que le biais dans l'échantillon serait secondaire par rapport à une nouvelle tendance qui se manifesterait par une autonomie sexuelle revendiquée par les filles québécoises, à la suite des transformations de la sphère sociosexuelle contemporaine parmi les jeunes adultes du Québec. Certains indices nous sont fournis par les recherches portant sur les attitudes sexuelles des étudiantes universitaires francophones qui sont plus permissives que les hommes. Elles sont aussi plus égalitaires que les hommes sur le plan des rôles sexuels (Lévy et al., 1993) et ont de plus des attitudes plus favorables à la cohabitation, qui apparaît comme un substitut acceptable au mariage (Hobart, 1992; Bibby, Posterski et Donald, 1992). Ces tendances suggèrent que les femmes ne se limiteraient plus à demander une égalité, mais 
qu'elles se revendiquent dans des conduites sexuelles affirmées. En ce sens, si ces résultats devaient être confirmés par d'autres études, ils indiqueraient une modification du double standard sexuel qui favorisait généralement plus l'expression de la sexualité chez les garçons. Cet indice, encore ténu, s'inscrirait dans la modernisation accélérée des conduites sexuelles au Québec.

Comme dans les autres recherches, l'âge reste un déterminant important d'une entrée dans la vie sexuelle tant pour l'ensemble de l'échantillon que pour chacun des genres pris séparément, confirmant qu'une telle éventualité s'accroît avec l'âge (Christopher et Roosa, 1991; Dorius, Heaton et Steffen, 1993). Parmi les garçons, le niveau scolaire constitue un déterminant significatif distinct de l'âge. Les répondants qui sont plus avancés dans le niveau d'études sont plus enclins à être actifs sexuellement, ce qui pourrait s'expliquer par le contexte sociosexuel propre au cégep. La première année de cégep, phase de transition par rapport au secondaire, nécessiterait pour les garçons une adaptation au rythme de travail, l'apprentissage de l'autonomie dans les études et le développement de nouveaux réseaux de pairs et ce, au dépens de la sociabilité sociosexuelle qui se voit repousser aux années suivantes. Il se pourrait aussi que la permissivité des pairs soit alors plus marquée, ce qui permettrait une expression sexuelle plus directe.

Parmi les filles, ce facteur n'intervient pas. En revanche, le profil d'études est significatif. Ainsi, celles qui sont inscrites dans le profil technique sont significativement plus enclines à être impliquées sexuellement que celles qui sont inscrites dans le profil général. Cela indiquerait peut-être une maturation sexuelle et relationnelle plus rapide parmi celles qui choisissent la filière technique, qui débouche plus directement sur le marché du travail. Le choix précoce d'une carrière, indicatif d'une quête d'autonomie et d'aspirations professionnelles plus définies, pourrait être associé à une autodétermination sexuelle plus avancée, ce qui ne serait pas le cas chez celles qui privilégient la filière universitaire, retardant ainsi leur entrée dans la vie sexuelle. Comme le suggèrent quelques études, la quête d'une réussite scolaire et l'ampleur des projets de scolarisation sont inversement corrélées à l'entrée dans la vie sexuelle (Miller et Sneeby, 1988), et cela semble se confirmer dans le cas de ces étudiantes. Le travail pendant les études - un facteur qui joue à la fois pour l'échantillon global et pour les garçons et les filles séparément - contribuerait à amplifier l'autonomie sur le plan sexuel. Ainsi, l'indépendance financière relative qui accompagne le travail, 
mais aussi les répercussions des expériences vécues dans un milieu professionnel, peuvent contribuer à diminuer le contrôle des agents de socialisation sur la prise des décisions concernant la vie sexuelle. Comme le montrent d'autres études (Forste et Heaton, 1988; Leigh, Weddle et Loewen, 1988; Miller et Bingham, 1989), la nature du contexte familial intervient aussi. Ainsi, ceux qui vivent dans le cadre d'une famille nucléaire sont plus enclins à ne pas être impliqués sexuellement, ce qui peut être lié à un contrôle plus grand des activités des jeunes adultes de la part des parents ou à la présence de modèles plus conservateurs de conduites sexuelles.

L'implication sexuelle est, par contre, nettement plus élevée parmi les Québécois francophones de souche. Cette distribution confirme les résultats d'autres recherches (Maticka-Tyndale et Lévy, 1992) qui montrent, en particulier, que les jeunes filles appartenant à ces groupes - où elles sont considérées comme les porteuses de la transmission de la culture de leur groupe - sont soumises à un contrôle sociosexuel sévère qui rejoint les idéaux de virginité dominants. Ces exigences socioculturelles contribuent à retarder l'entrée dans la vie sexuelle. La pratique religieuse, l'un des facteurs le plus souvent mentionnés dans la nonimplication sexuelle (Forste et Heaton, 1988 ; Miller et Bingham, 1989), apparaît aussi dans notre étude comme l'un des déterminants de ce statut, malgré la baisse significative des pratiques religieuses au Québec. Celles-ci, associées généralement à une forte religiosité et à des attitudes plus restrictives face à la sexualité à cause des codes religieux qui freinent son expression en dehors des liens de mariage, continuent de moduler l'entrée dans la vie sexuelle. Il en est de même quant au type de collège, public ou privé. Ainsi, les élèves qui fréquentent les collèges privés sont plus enclins à ne pas s'impliquer sexuellement. Les deux types d'établissements se différencient sans doute à la fois sur le plan des concentrations de programmes, des mécanismes de recrutement et des catégories socioculturelles d'où proviennent les élèves qui les fréquentent. Le contexte collégial et familial peut être aussi plus sévère pour ce groupe de jeunes adultes, d'où un contrôle plus fort sur la sexualité.

\section{Déterminants psychosociaux}

Un trait de personnalité, la domination, semble caractériser davantage les répondants impliqués sexuellement, ce qui peut être lié à la capacité d'affirmation de soi. Ce trait est marquant 
chez les garçons, mais non chez les filles. Il en est de même de la quête de sensations fortes, qui est l'un des déterminants significatifs de l'entrée dans la vie sexuelle. Comme l'ont montré les travaux de Zuckerman (1994), les individus en quête de sensations fortes ont tendance à rechercher des expériences sexuelles variées et multiples. Nos résultats suggèrent que cette quête peut intervenir sur les décisions entourant le début de la vie sexuelle. Parmi les filles seulement, une conception de la sexualité basée sur le partage semble prévaloir chez les répondantes actives sexuellement. Cette constatation rejoint celles des études qui montrent que la communication et le contexte affectif sont essentiels à l'expression de la sexualité chez les femmes (Christopher et Roosa, 1991), mais ils joueraient un rôle plus secondaire chez les garçons. Des attitudes égalitaires vis-à-vis des rôles sexuels semblent aussi l'un des déterminants de l'activité sexuelle, tant pour les garçons que pour les filles. Ces résultats suggèrent que la modernisation des attitudes quant à la place des femmes dans le contexte social interviendrait aussi sur la sphère sexuelle et contribuerait à favoriser une implication sexuelle plus grande. Il en est de même lorsque les individus sont capables de contrôler l'expression de leur plaisir. Le contrôle par la volonté de l'expression sexuelle discrimine entre les garçons actifs et non actifs sexuellement, ce qui n'intervient pas chez les filles. Les attitudes à l'égard du préservatif plus négatives parmi les filles impliquées sexuellement suggèrent que l'expérience sexuelle contribue à modifier les perceptions entourant cet objet à cause des contraintes concrètes qu'il impose à la vie sexuelle. Les perceptions du contrôle de l'usage du préservatif rejoignent en partie cette hypothèse. En effet, ce contrôle dans la relation est perçu comme plus faible chez les répondants actifs sexuellement qui rapportent aussi plus de gêne, tant chez les garçons que chez les filles. À l'image idéale et théorique qui semble dominer chez les non-actifs vient se substituer une perception plus réaliste de son usage chez les autres répondants. En dernier lieu, la peur d'une grossesse constitue l'un des déterminants significatifs dans les scénarios des filles sexuellement non actives. Par contre, cette peur se retrouve face aux MTS-sida chez les garçons, mais non chez les filles. Ces résultats, dans leur ensemble, confirment les hypothèses de Jedlicka et Robinson (1987) voulant que la peur de la grossesse soit l'un des facteurs saillants dans la décision de repousser l'entrée dans la vie sexuelle. Cependant, contrairement à leurs conclusions, la peur face aux MTS-sida est l'un des modulateurs de l'expression sexuelle chez 
les garçons. Ces différences dans les peurs rattachées à la vie sexuelle - la grossesse pour les filles et les MTS-sida pour les garçons - laissent supposer que, malgré les transformations des modèles sexuels, la sexualité féminine est encore marquée par les préoccupations liées à la reproduction lorsqu'il s'agit de franchir le pas vers une sexualité plus active, une problématique absente chez les garçons qui sont plus enclins à considérer les risques personnels des MTS et du sida. Par contre, lorsqu'on considère la perception des risques, une autre configuration apparaît. Pour les garçons et les filles, la perception des risques d'être confrontés à une grossesse intervient chez les élèves impliqués sexuellement, mais les risques de contracter une MTS ou le sida sont l'un des déterminants chez les filles, mais non parmi les garçons qui sont actifs sexuellement. Cette situation suggère que les filles, une fois sexuellement actives, ont à assumer un double ensemble de préoccupations, ce qui n'est pas le cas pour les garçons. Un double standard demeure donc présent dans ce domaine.

Cet ensemble de données peut aider à orienter les plans d'intervention psychosociale auprès des cégépiens francophones. Ainsi, il serait possible pour les éducateurs et les travailleurs sociaux d'élaborer des programmes de réflexion et de sensibilisation portant sur la transition à une vie sexuelle active, en particulier chez les sujets les plus jeunes et inscrits en première année de cégep. Une attention particulière doit être aussi portée à la présence des élèves provenant de groupes ethnoculturels spécifiques ou plus religieux et dont les modèles sociosexuels peuvent les mettre en conflit avec ceux, dominants, dans la société québécoise. Les tensions intrafamiliales, de même que les pressions des pairs, peuvent créer des stress importants dont les répercussions doivent être prises en considération dans les approches d'intervention. Les différences dans les profils des garçons et des filles, de même qu'entre les collèges publics et privés, suggèrent aussi l'importance de spécifier ces approches en fonction des écosystèmes dans lesquels ces individus s'insèrent. Ces stratégies plus adaptées aux différents milieux permettront de mieux atteindre et de conseiller les élèves dans leur développement psychosexuel. De ce point de vue, l'analyse des déterminants suggère qu'il est important de travailler autour des thèmes qui touchent la recherche de sensations fortes et leurs conséquences sur la prise de risques face aux MTS et au sida. Associé à cette question, le travail sur les perceptions à l'égard du préservatif et de son usage constitue à notre avis un 
axe essentiel de sensibilisation. Les attitudes vis-à-vis du préservatif, les perceptions du contrôle de son usage dans la relation et la gêne à son égard sont, nous l'avons vu, problématiques chez les répondants qui sont impliqués sexuellement. De ce fait, il est important d'assurer que le passage à une vie sexuelle active et aux ajustements qu'elle nécessite ne provoque pas une baisse ou un rejet des pratiques préventives nécessaires dans le contexte créé par les MTS-sida. À cet égard, une attention particulière doit être portée aux perceptions croisées des garçons et des filles quant aux peurs et aux risques entourant la grossesse et les MTSsida, afin d'assurer une meilleure compréhension de ces facteurs dans la dynamique entourant les relations sexuelles.

\section{CONCLUSION}

Bien que cette recherche ne porte pas directement sur les populations desservies par les intervenants en service social, elle peut les aider, de même que les sexologues, intervenants en santé et médecins ouvrant auprès des adolescents et jeunes adultes, à mieux comprendre les configurations sociosexuelles des cégépiens francophones. Parmi eux, environ $25 \%$, en particulier les garçons, n'ont pas encore eu d'activité sexuelle de type génital, ce qui s'explique par des facteurs liés à la fois à l'âge et au contexte collégial, familial, ethnoculturel et religieux. Ces paramètres importants sont relativement semblables pour les garçons et les filles. Sur le plan des déterminants psychosociaux de l'implication sexuelle, des convergences apparaissent dans les scénarios des garçons et des filles, mais ils diffèrent aussi en particulier en ce qui a trait aux perceptions du contrôle de la sexualité, des attitudes à l'égard du préservatif ainsi que des peurs et des risques entourant les MTS-sida. Ces données peuvent orienter l'intervention en soulignant l'importance de faire porter l'attention sur les écosystèmes dans lesquels s'insèrent ces élèves ainsi que leurs valeurs culturelles et religieuses. Il semble aussi essentiel de réfléchir sur des programmes portant sur les phases de transition à une vie sexuelle active susceptibles de jouer de façon significative sur la mise en place des stratégies de prévention face à la grossesse et aux MTS-sida et ce, en tenant compte des différences de genre qui continuent d'exister aujourd'hui et dont il convient de préciser l'ampleur et le retentissement sur la vie sexuelle des jeunes adultes. 


\section{Références bibliographiques}

Bibby, R., W. Posterski et C. Donald (1992). Teen Trends. A nation in motion. Toronto: Stoddart, 344 pages.

BILLY, J. et J.R. UDRY (1985). "The influence of male and female best friends on adolescent sexual behavior», Adolescence, vol. 20 : 21-32.

CARroll, L., D.K. VOlK et S.J. HYde (1985). «Difference between males and females in motives for engaging in sexual intercourse», Archives of Sexual Behavior, vol. $14: 131-139$.

CHILMAN, C. (1980). "Social and psychological research concerning adolescent childbearing : 1970-1980», Journal of Marriage and the Family, p. 793-805.

ChIlman, C. (1983). Adolescent Sexuality in a Changing American Society: Perspectives for the human services professions. New York: John Wiley and Sons, Inc., 334 pages.

CHRISTOPHER, F.S. et M.W. RoOSA (1991). «Factors affecting sexual decisions in the premarital relationships of adolescents and young adults ", dans Sexuality in close relationships, sous la direction de Susan Sprecher. New Jersey: Lawrence Erbaum Associates, p. 111-135.

Christopher, F.S. et R.M. CATE (1984). «Factors involved in premarital sexual decision-making ", Journal of Sex Research, vol. 20:363-376.

CHRISTOPHER, F.S. et R.M. CATE (1988). «Premarital sexual involvement: A developmental investigation of relational correlates », Adolescence, vol. 23 : 793-803.

Cverkovich, G et B. Grote (1980). «Psychological development and the social problems of teenage illegitimacy», dans Adolescence pregnancy and chilbearing: findings from research, sous la direction de C. Chilman. DHHS Publications (NIH) 81-2077, U.S. Washington, DC: Government Printing Office, p. 15-41.

Cverkovich, G., B. Grote, E. Lieberman et W. Miller (1978). «Sex role development and teenage fertility-related behavior », Adolescence, vol. $13: 231-236$.

Delamater, J. (1981). "The social control of sexuality », Annual Review of Sociology, vol. $7: 263-290$.

DRYFoOs, J. (1982). "The epidemiology of adolescent pregnancy: incidence, outcomes, and interventions », dans Pregnancy in adolescence: needs, problems and management, sous la direction de I. Stuart et C.Welts. New York: Van Norstrand Reinhold Co., p. $27-47$.

FisHer, T. (1989). "Family sexual communication and adolescent sexual behavior», Journal of Marriage and the Family, vol. 51:637-639.

FLEWELLING, R.L. et K.E. BAUMAN (1990). "Family structure as a predictor of initial substance use and sexual intercourse in early adolescence », Journal of Marriage and the Family, vol. 52 : 171-180.

Forste, R.T. et T.B. HEATON (1988). «Initiation of sexual activity among female adolescents », Youth and Society, vol. 19, $\mathrm{n}^{\circ} 3: 250-268$. 
Furstenberg, F., K.A. Moore et J.L. Peterson (1985). «Sex education and sexual experience among adolescents », American Journal of Public Health, vol. 75 : 1331-1332.

Furstenberg, F., R. HerCeg-BAron, J. SheA et D. Webb (1986). «Family communication and contraceptive use among sexually active adolescents» dans School-age pregnancy and parenthood: Biosocial dimension, sous la direction de J.B. Lancaster et B.D. Hamburg. Hawthorne, NY : Aldine de Gruyter, p. 251-261.

Gullotta, T., G.R. AdAms et R. Montmayer (1993). Adolescent sexuality. Newbury Park: Sage Publications, 217 pages.

HARRIS, L. et al. (1986). American teens speak: sex, myths, TV, and birth control. New York: Louis Harris and Associates.

HAYES, C.D. (1987). Risking the future: Adolescent sexuality pregnancy, and childbearing. Washington, D.C.: National Academy Press, 337 pages.

Hobart, C.W. (1992). «How they handle it. Young Canadians, Sex, and Aids », Youth and Society, vol. 23, nº 4: 411-433.

HOFFERTH, S. (1987). "Factors affecting initiation of sexual intercourse » dans Risking the future: Adolescent sexuality pregnancy, and childbearing, sous la direction de S.L. Hofferth et C.D. Hayes. Washington, DC: National Academy Press, p. 7-35.

JEDLICKA, D et I.E. RoBINSON (1987). «Fear of veneral disease and other perceived restraints on the occurrence of premarital coitus », Journal of Sex Research, vol. 23 : 391-396.

KAHN, J., K.W. SMITH et E.J. ROBERTS (1984). «Family communication and adolescent sexual behavior.» Unpublished manuscript. Cambridge, MA : American Institute for Research.

LEIGH, G.K., K.D. WedDle et I.R. LOEWEN (1988). "Analysis of the timing of transition to sexual intercourse for black adolescent females ", Journal of Adolescent Research, vol. 3 : 333-344.

LÉvy, J.J., A. Dupras, J.-M. SAMSON, P. CAPpon, L.-R. Frigault, A.-M. LAROSE (1993). Facteurs de risque face au sida et comportements sexuels des étudiant(e)s universitaires de Montréal. Montréal: Université du Québec à Montréal, Département de sexologie.

MATICKA-TYNDALE, B. et J.J. LÉVY (1992). Sexualité, contraception et sida chez les jeunes adultes: Variations ethno-culturelles. Montréal: Éditions du Méridien.

LÉVY, J.J et D. SANSFAÇON (1994). "Les orientations sexuelles », dans Problèmes sociaux du Québec, sous la direction de F. Dumont, S. Langlois et Y. Martin. Institut québécois de recherche sur la culture, p. 455-473.

LÉVY, J.J., J. OTIS et J.-M. SAMSON (1996). Risques face au sida, relations de pouvoir et styles de communication sexuelle chez les étudiants des cégeps francophones du Québec. Rapport de recherche.

Miller, B. et C.R. BINGHAM (1989). «Family configuration in relation to the sexual behavior of female adolescents ", Journal of Marriage and the Family, vol. $51: 499-506$. 
Miller, B. et K.R. SNEeby (1988). "Educational correlates of adolescents' sexual attitudes and behavior », Journal of Youth Adolescence, vol. $17: 521-530$.

Miller, B. et T.D. OlSEN (1988). «Sexual attitudes and behavior of high school students in relation to background and contextual factors ", Journal of Sex Research, vol. 24 : 194-200.

Miller, B., J. CARD, R. PAikoff et J. Peterson (1992). Preventing adolescent pregnancy. Newbury Park, Sage Publications. 296 pages.

Miller, B., J.K. MCCOY, T.D. Olsen et C.M. WAllaCe (1986). "Parental discipline and control attempts in relation to adolescent sexual attitudes and behavior ", Journal of Marriage and the Family, vol. $48: 503-512$.

Miller, B., R. Christensen et T.D. Olsen (1987). "Self-esteem in relation to adolescent sexual attitudes and behavior», Youth and Society, vol. $18: 93-111$.

MOORE, K. (1990). «Adolescent sexual behavior, pregnancy, and parenting: Research through the 1980s ", Journal of Marriage and the Family, vol. 52: 1025-1044.

Moore, K., M.C. SimMs et C.L. Betsey (1986). Choice and Circumstance. New Brunswick, NJ : Transaction Books.

OTIS, J. (1996). Santé sexuelle et prévention des MTS et de l'infection au VIH. Bilan d'une décennie de recherche au Québec auprès des adolescents et adolescentes et des jeunes adultes. Bibliothèque nationale du Canada, 164 pages.

OTIS, J., G. GODIN et J. LAMBERT (1993). Motivational factors towards a choice for prevention of HIV infection among adolescents : continence or condom uses. $9^{\mathrm{e}}$ Conférence internationale sur le sida, Berlin.

PROPPER, S. et R.A. BROWN (1986). «Moral reasoning, parental sex attitudes, and sex guilt in female college students », Archives of Sexual Behavior, vol. $15: 331-340$.

RoDGERs, J. et D. Rowe (1988). "Influence of siblings on adolescent sexual behavior", Developmental Psychology, vol. 24, $\mathrm{n}^{\circ} 5$ : 722-728.

RODGERS, J. et D.C. Rowe (1990) «Adolescent sexual activity and mildly deviant ", Journal of Family Issues, vol. 2 : 274-293.

ROSEN, R, L. HERSKOVITZ et J. STACK (1980). «The first sexual experience: preliminary findings for a group of pregnant teens », Abstracts no. 1505, dans Sociological Abstracts Supplements no. 103. Abstracts of papers presented at the Annual Meeting of the Society for the Study of Social Problems, p. 107.

Russ-EFT, D., M. SPRENGER et A. BEever (1979). «Antecedents of adolescent parenthood and consequences at age $30 »$, Family Coordinator, vol. $28: 173-178$.

SANTÉ QUÉBEC (1992). Enquête québécoise sur les facteurs de risque associés au sida et aux autres MTS : la population des 15-29 ans. Québec: Gouvernement du Québec, MSSS. 
SMITH, M.U. et al. (1993). Psychological, sociological and demographic correlates of unsafe sexual behavior among students (aged 14-16) in Georgia (USA). 9e Conférence internationale sur le sida, Berlin.

STEWART, D.C. (1987). "Sexuality and the adolescent: Issues for the clinician ", Primary Care, vol. 14 : 83-99.

STROUSE, J.S. et R.A. FABES (1987). «A conceptualization of transition to nonvirginity in adolescent females », Journal of Adolescent Research, vol. 2 : 331-348.

SugAr, M. (1990). A typical adolescence and sexuality. Sous la direction de Max Sugar. New York: W.W. Norton, 215 pages.

TABLE DE CONCERTATION SUR LA SANTÉ ET LA SEXUALITÉ DE LA COMMISSION SCOLAIRE LA NEIGETTE (1995). "Étude sur les connaissances et les comportements concernant la sexualité, les maladies transmissibles sexuellement et le sida chez les élèves de $1^{\text {re }}$ et $2^{\mathrm{e}}$ secondaire de la commission scolaire La Neigette. » Synthèse des résultats de l'enquête. Document non publié, 29 pages.

Thornton, A. et D. CAMBURn (1987). "The influence of the family on premarital sexual attitudes and behavior», Demography, vol. 24 : 323-340.

Treboux, D. et N.A. BusCH-Rossnagel (1990). "Social network influences on adolescent sexual behaviors", Journal of Adolescent Research, vol. $5: 175-189$.

WEINSTEIN, M. et A.L. ThORNTON (1989). "Mother-child relations and adolescent sexual attitudes and behavior», Demography, vol. 26 : 563-577.

Whitbeck, L.B., D.R. HOYT, M. Miller et M.-Y. KAO (1992). «Parental support, depressed affect, and sexual experience among adolescents », Youth and Society, vol. 24, n 2 : 166-177.

White, S.D. et R. DeBlassie (1992). «Adolescent sexual behavior», Adolescence, vol. 27 : 183-191.

Young, E.W., L.C. Jensen, J.A. Olsen et B.P. CUNDICK (1991). «The effects of family structure on the sexual behavior of adolescents », Adolescence, vol. 26: 977-986.

ZELNIK, M. et F.K. SHAH (1983). «First intercourse among young Americans », Family Planning Perspectives, vol. $15: 64-70$.

ZELNIK, M. et J. KANTNER (1980). «Sexual activity, contraceptive use and pregnancy among metropolitan area teenagers: 1971-1979», Family Planning Perspectives, vol. $12: 230$.

ZELNIK, M. et Y. KIM (1982). "Sex education and its association with teenage sexual activity, pregnancy and contraceptive use », Family Planning Perspectives, vol. 14 : 117-126.

ZELNIK, M., J. KANTNER et K. FORD (1981). Sex and pregnancy in adolescence. Beverly Hills, CA : Sage Publications.

ZUCKERMAN, M. (1994). Behavioral Expressions and Biosocial Bases of Sensation Seeking. New York: Cambridge University Press, 211 pages. 